\title{
Bilateral proptosis: an unusual primary presentation of scurvy-a case report
}

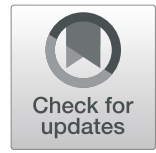

Damini S. ${ }^{*}$, Rashmi Dixit ${ }^{1}$, Tamanna Khullar ${ }^{1}$ and K. Rajeshwari ${ }^{2}$

\begin{abstract}
Background: Scurvy is a nutritional vitamin C deficiency disease which shows classical signs on radiographs most commonly along long bones with subperiosteal hemorrhage being a classical finding. Primary presentation of scurvy with orbital hemorrhage presenting as proptosis is however uncommon.

Case presentation: We report an unusual primary presentation of scurvy in a 4-year-old boy with West syndrome and global developmental delay who presented to the emergency with complaints of bilateral proptosis and eyelid swelling (right > left) which on examination revealed bluish discolouration of bilateral eyelids. On further multimodality imaging investigation, orbital subperiosteal hemorrhages were identified as the cause, along with classical radiographic features of scurvy in bilateral lower limb long bones. The diagnosis was further bolstered by rapid resolution of periorbital ecchymosis after initiating oral vitamin C supplementation.

Conclusion: To the best of our knowledge, primary presentation of scurvy in the form of bilateral proptosis due to orbital hemorrhages although uncommon can still be a manifestation of scurvy in addition to the more routinely detected subperiosteal hemorrhages along long bones. The significance of establishing the aetiology in such cases of orbital hemorrhage cannot be overemphasised, in view of drastic clinical recovery after starting vitamin $C$ therapy.
\end{abstract}

Keywords: Scurvy, Subperiosteal hematoma, Proptosis, Racoon eyes, Periorbital ecchymosis, Vitamin C deficiency

\section{Background}

Periorbital swelling associated with bluish discoloration is referred to as periorbital ecchymosis or raccoon eye. It is a manifestation of contusion and stretching of capillaries leading to rupture and tracking of blood into the periorbital tissues, causing blue or purple discolouration of the eyelids [1]. It is commonly the telltale sign of head and neck trauma including skull base fractures, soft tissue injuries and facial fractures with resultant hemorrhage [1].

Non-traumatic periorbital ecchymosis is relatively uncommon. It is usually due to preexisting lesions such as metastasis from neuroblastoma, leukaemia or Ewing's sarcoma in children. Ecchymosed orbital proptosis

\footnotetext{
* Correspondence: damy2804@gmail.com

'Department of Radiodiagnosis, Maulana Azad Medical College and Lok

Nayak Hospital, Bahadur Shah Zafar Marg, New Delhi 110002, India

Full list of author information is available at the end of the article
}

(raccoon eyes) is considered the hallmark of neuroblastoma metastasis in children [2] and is thought to occur due to obstruction of the palpebral vessels in and around the orbits by the metastatic tissue [3].

In adults, migraine, amyloidosis, leukaemic deposits or multiple myeloma lesions are few common causes of periorbital ecchymosis apart from Valsalva manoeuvre which lead to increased venous pressure $[1,4]$. Underlying coagulopathy and bleeding diathesis, vascular malformations and iatrogenic causes can also lead to orbital hemorrhages [4].

Scurvy is a clinical syndrome with manifestations due to severe prolonged vitamin $C$ deficiency. The vitamin $C$ deficiency is usually either due to poor diet or poor food preparation such as unfortified boiled milk. Low plasma levels of vitamin $\mathrm{C}$ are also seen in children with iron overload, despite the absence of poor dietary intake. This may be due to reduced gastrointestinal absorption along 
with increased catabolism of ascorbate [5]. Children with mental or physical disabilities are more prone to develop scurvy [6]. Vitamin $C$ deficiency leads to weakening of supporting tissue of mesenchymal origin such as collagen in capillary walls and skin, osteoid in bone, chondroid in cartilage and dentin in teeth. The common clinical presentations include irritability, pain, tenderness, pseudo paralysis of the limbs and bleeding gums. Hemorrhage in scurvy occurs owing to progressive vascular fragility caused by deficient collagen synthesis in basement membrane of blood vessels [6].

Subperiosteal hemorrhage is a well-known manifestation of scurvy, commonly seen along the long bones. In 1905, Snow documented bleeding into the subperiosteal space along orbital roof in the post-mortem findings of an infant who had expired from scurvy [7]. In the nineteenth century, infantile scurvy became rampant as infants were being fed with sterilised infant formula or boiled milk which was deficient in vitamin $C$ [8]. Though scurvy was historically a significant cause of infantile orbital hemorrhage, it is seldom seen nowadays, even in developing countries. Also, up to $80 \%$ of scurvy patients clinically present with musculoskeletal symptoms; in contrast, initial orbital presentation is relatively rare and seldom reported in literature [6].

Classical radiographic findings in the appendicular skeleton in scurvy include linear area of increased density in physis corresponding to normal mineralisation activity in the zone of provisional calcification (Frankel's line) along with adjacent horizontal bands of radiolucency corresponding to reduced osteoblastic activity leading to reduced trabecular bone mass (Trummerfelds' zone) [9]. Extension of zone of provisional calcification beyond the metaphyseal margins causing periosteal elevation and spur formation is known as Pelkan spurs [9, 10]. Sharply marginated epiphysis by a sclerotic rim with central lucent area gives rise to Wimberger ring sign which is due to reduced cartilage proliferation with unimpaired mineralisation (sclerosis) [9, 10]. Classically, soft tissue swellings with periosteal elevation along diaphysis of long bones are seen due to subperiosteal hemorrhages $[9,11]$. The constellation of radiographic findings, as seen in our case, is highly suggestive of scurvy.

Orbital hemorrhages in scurvy are typically superior and subperiosteal in location [12], which is similar to the findings in our case. As the patient present with rapidly worsening symptoms in a short duration, orbital abscess and malignancies such as small round blue cell tumours (neuroblastoma, rhabdomyosarcoma, leukaemia, lymphoma) which are the common cause of spontaneous orbital swelling in children need to be ruled out, necessitating orbital imaging $[4,13]$.

\section{Case presentation}

A 4-year-old male child with global developmental delay and West syndrome which is characterised by classical triad of infantile spasms, hypsarrhythmia and developmental arrest or regression [14] was brought to the emergency with complaints of right eye proptosis and eyelid swelling for 10 days and left eyelid swelling for a week. There was no history of trauma, surgery, insect bite or any history suggestive of non-accidental trauma. There was no history of similar complaints in the past.

On examination, there was periorbital swelling with slight bluish discolouration around the eyes, especially on the right, and limitation of movement in the right eye. There were no other mucocutaneous lesions elsewhere in the body. In view of periorbital swelling associated with bluish discolouration, skull radiograph (Fig. 1) followed by computed tomography (Fig. 2) of the head without contrast was performed which revealed no evidence of any skull base fracture or intracranial bleed. The soft tissue window CT images revealed heterogeneously mildly hyperdense soft tissue like lesions in bilateral superior extraconal spaces (right $>$ left) needing further characterization. The patient was then referred to the paediatric department for detailed evaluation.

General physical examination revealed microcephaly, high-arched palate, coarse facies and pallor. Local examination revealed right eye swelling and restricted ocular movement, conjunctival chemosis, exposure keratitis and ecchymosis in both eyelids. Patient had poor oral hygiene with history of bleeding gums intermittently. The CNS examination revealed bilateral upgoing plantar reflexes; the tone was increased in both limbs with their power was 2/5. Deep tendon reflexes were present. The patient also had tender lower limbs and a limp. However, no obvious discolouration or open wounds were seen at the lower limbs. The child was not breast fed and had a predominant milk-based diet. The rest of systemic examination revealed no significant abnormality.

Laboratory investigations revealed anaemia (hemoglobin $6.3 \mathrm{mg} / \mathrm{dl}$ ) with a normal total white blood cell count $\left(10.3 \times 10^{9} / \mathrm{L}\right)$, platelet count $\left(2.52 \times 10^{5} / \mathrm{uL}\right)$ and coagulation profile (PT 12.5s, INR 1.0). The liver function tests (total protein $7.2 \mathrm{~g} / \mathrm{dL}$, serum albumin 3.4 $\mathrm{g} / \mathrm{dL}$, ALP $95 \mathrm{IU}$ ) and kidney function tests (blood urea $22 \mathrm{mg} / \mathrm{dL}$, serum creatinine $0.22 \mathrm{mg} / \mathrm{dL}$ ) were within normal limits.

In view of the periorbital swelling with bluish discolouration, no evidence of fracture on cranial CT scan and extraconal heterogeneously mildly hyperdense lesions, the patient was referred for ultrasound of the abdomen to rule out abdominal mass lesion such as neuroblastoma. Ultrasound abdomen did not reveal any abnormality. An MRI examination of orbit and brain was also 


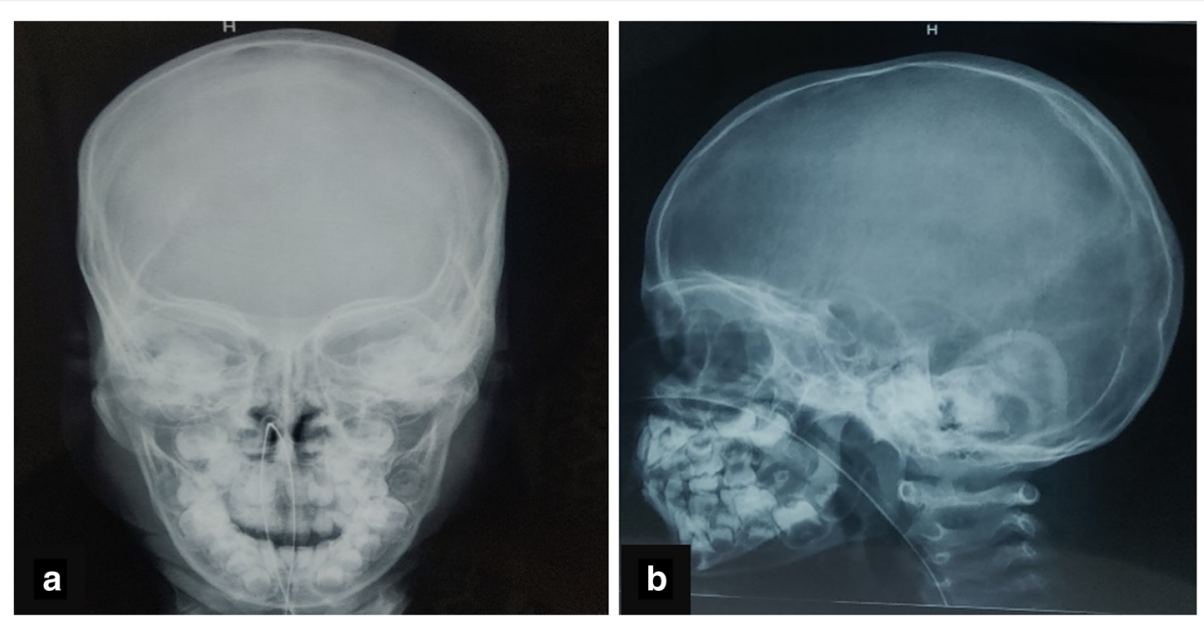

Fig. 1 a, b Posteroanterior and lateral radiograph of the skull reveals no evidence of fracture. Nasogastric tube in situ

requested to exclude possibility of intraorbital mass lesion or metastasis.

MRI (Fig. 3) showed smoothly marginated collections in extraconal location along the roof of both orbits, displacing superior recti muscle inferiorly with maintained intervening fat planes. They appeared uniformly hyperintense on T1W, T2W and FLAIR images without any signal suppression on fat-saturated images. Findings suggested subperiosteal hematomas. Both globes, extraocular muscles and optic nerves, appeared normal in morphology and intensity. No intraorbital soft tissue mass lesion was noted.

Radiographs of both knee joints (Fig. 4) were also done to evaluate the cause for his limp. They revealed dense metaphyseal bands at both distal femoral physes with adjacent proximal horizontal bands of radiolucency suggestive of zone of provisional calcification with Trummerfeld zones. Mild flaring along metaphyseal corners was noted at distal femoral metaphysis suggestive of
Pelkan spurs. Distal femoral epiphyses appeared centrally lucent with sharply marginated sclerotic rim giving the Wimberger ring sign. Similar findings were also seen at both proximal tibial physes. The findings were highly suggestive of scurvy. However, there was no periosteal elevation or soft tissue opacities along diaphysis of long bones.

Based on the constellation of leg pain, bleeding gums, dietary history, mentally challenged condition along with lower limb radiographic findings and orbital hemorrhages confirmed on MRI, a final diagnosis of scurvy was made.

The patient could not afford serum ascorbic acid levels; however, the child was administered vitamin $\mathrm{C}$ empirically following which a drastic improvement was observed in the clinical condition of the child. Rapid clinical recovery with vitamin C-based treatment was used as surrogate diagnostic confirmation criteria. Following a week of treatment, left orbital USG revealed
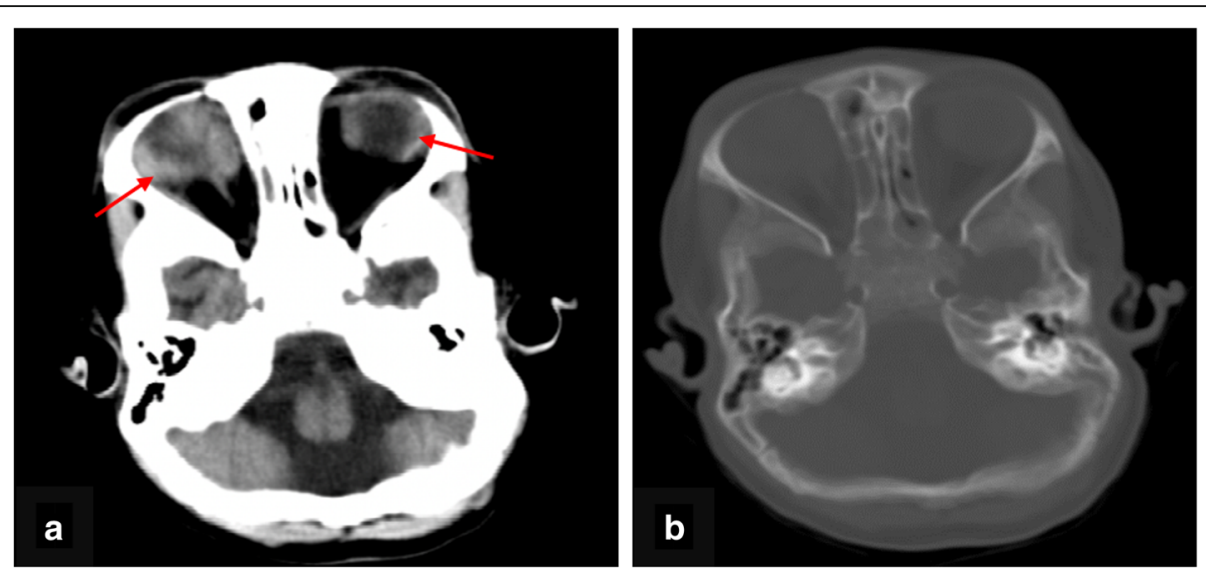

Fig. 2 a, b Axial section of NCCT head in soft tissue and bone window confirms absence of fracture and heterogeneously hyperdense extraconal content, more on right side as compared to left (arrows) 

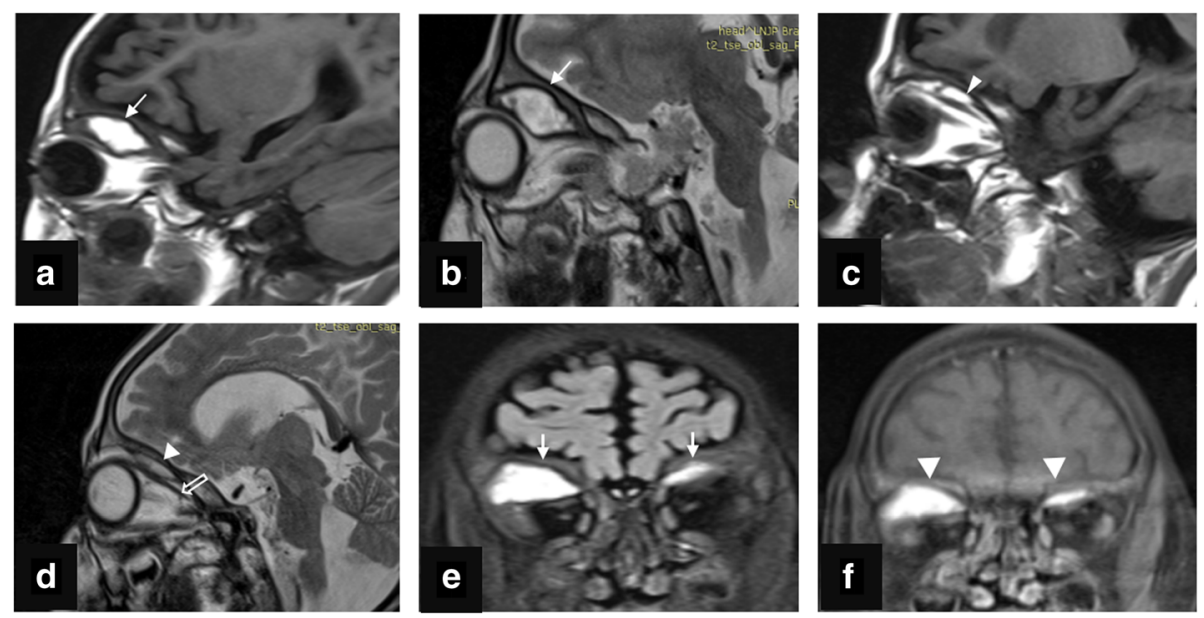

Fig. 3 a, b T1 and T2w sagittal images of right orbit reveal sharply marginated T1 and T2W hyperintense collection (arrows) along orbital roof with resultant downward displacement of superior rectus muscle. c, d T1- and T2-weighted sagittal images of the left eye reveal a similar smaller collection appearing hyperintense on T1 and T2W images (white arrowheads) along orbital roof with resultant downward displacement of superior rectus muscle (open arrow). e, f FLAIR coronal and T1 fat-suppressed images reveal the collections along bilateral orbital roof appearing hyperintense on FLAIR (arrows) with no signal suppression on fat-suppressed images (arrowheads)

complete resolution of the collection while right orbital USG revealed reduction in the size of the collection appearing heterogeneously hypoechoic with fine internal moving echoes, superior to the right globe.

\section{Conclusions}

In the absence of ascorbic acid level assay, diagnosis of scurvy is primarily based on clinical as well as radiological features. Primary presentation with orbital hemorrhage, although uncommon, can still be a manifestation of scurvy in addition to the more routinely detected subperiosteal hemorrhages along long bones. The diagnosis of scurvy is often overlooked due to its relative rarity in occurrence. The significance of establishing the aetiology in cases of orbital hemorrhage cannot be overemphasised, in view of drastic clinical recovery after starting timely vitamin $\mathrm{C}$ therapy in cases of scurvy.

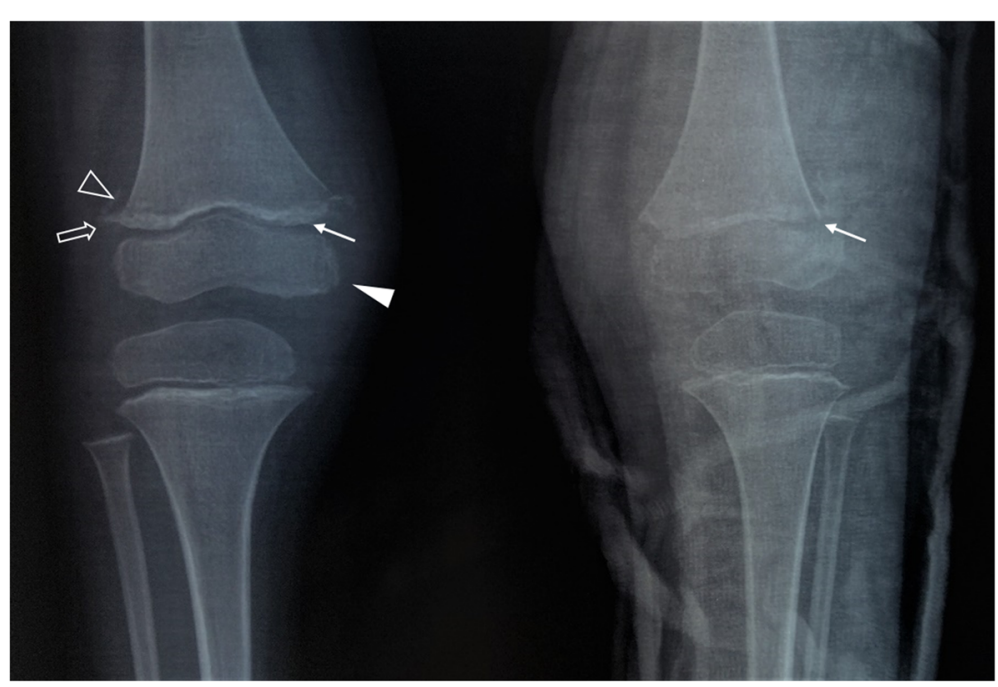

Fig. 4 Anteroposterior radiograph of knee joint reveals metaphyseal dense bands in bilateral distal femoral metaphyses (white arrows) with adjacent proximal horizontal bands of radiolucency (open arrowhead) suggestive of zone of provisional calcification with Trummerfeld zones. Flaring at metaphyseal corners of distal femoral metaphysis (open arrows) suggestive of Pelkan spurs. Centrally lucent epiphysis with sharply marginated sclerotic rim (white arrowhead) (Wimberger ring sign). Similar findings also seen in bilateral proximal tibial physes. The findings are characteristic for scurvy 


\section{Abbreviations}

ALP: Alkaline phosphatase; INR: International normalized ratio; MRI: Magnetic resonance imaging; PT: Prothrombin time; T1W: T1 weighted; T2W: T2 weighted; FLAIR: Fluid-attenuated inversion recovery; USG: Ultrasonography

\section{Acknowledgements}

Not applicable

\section{Authors' contributions}

DS obtained the patient data, analysed the images, and designed and drafted the manuscript. DS and RD revised the manuscript critically for important intellectual content. The authors read and approved the final manuscript.

\section{Funding}

None

Availability of data and materials

Not applicable

\section{Declarations}

\section{Ethics approval and consent to participate}

Not applicable

\section{Consent for publication}

Written informed consent was obtained from the parent of the patient for publication of this case report and accompanying images.

\section{Competing interests}

The authors declare that they have no competing interests.

\section{Author details}

${ }^{1}$ Department of Radiodiagnosis, Maulana Azad Medical College and Lok Nayak Hospital, Bahadur Shah Zafar Marg, New Delhi 110002, India. 2Department of Paediatrics, Maulana Azad Medical College and Lok Nayak Hospital, Bahadur Shah Zafar Marg, New Delhi 110002, India.

Received: 19 April 2021 Accepted: 3 July 2021

Published online: 20 July 2021

\section{References}

1. Nasiri J, Zamani F (2017) Periorbital ecchymosis (raccoon eye) and orbital hematoma following endoscopic retrograde cholangiopancreatography. Case Rep Gastroenterol 11(1):134-141. https://doi.org/10.1159/000456657

2. Büyükavci M, Yildirim ZK (2009) Recurrent bruising of lower eyelids: a presenting symptom of neuroblastoma in a child/Alt gözkapaklarinda tekrarlayan morluklar yakinmasiyla basvuran nöroblastomlu bir çocuk olgu. Turk J Haematol 26(4):210

3. Timmerman R (2003) Raccoon eyes and neuroblastoma. N Engl J Med 349(4):e4. https://doi.org/10.1056/ENEJMicm020675

4. McNab AA (2014) Nontraumatic orbital haemorrhage. survey of ophthalmology, 59(2): 166-184.

5. Roeser HP (1983) The role of ascorbic acid in the turnover of storage iron. Semin Hematol 20(2):91-100

6. Cheah SC, Tang P, Matthew TJ, Ooi MH, Husain S (2020) Spontaneous orbital haematoma in a scurvy child: a forgotten diagnosis. Int J Pediatr Otorhinolaryngol 137:110224. https://doi.org/10.1016/j.ijporl.2020.110224

7. Snow I (1905) Eye symptoms of infantile scurvy. A case of infantile scurvy with extreme protrusion of the right eyeball, shown by autopsy to be due to a large retrobulbar hematoma. Arch Pediatr 1905(22):576e80

8. Rajakumar K (2001) Infantile scurvy: a historical perspective. Pediatrics 108(4): e76-e76. https://doi.org/10.1542/peds.108.4.e76

9. Joshi R, Gustas-French CN, Fanburg-Smith JC, Helm KF, Flemming D (2019) Scurvy: a rare case in an adult. Skeletal Radiol 48(6):977-984. https://doi. org/10.1007/s00256-018-3069-3

10. Pelkan F (1925) The roentgenogram in early scurvy. Am J Dis Child 30(2): 174-188

11. Nelson WE, Doughty WM, Mitchell AG (1933) Roentgenographic visualization of subperiosteal hemorrhage in infantile scurvy. JAMA 101(1): 14-17. https://doi.org/10.1001/jama.1933.02740260016004
12. Sloan B, Kulwin DR, Kersten RC (1999) Scurvy causing bilateral orbital hemorrhage. Arch Ophthalmol 117(6):842-843. https://doi.org/10.1001/a rchopht.117.6.842

13. Corl K, Hofmann RJ, Sutton E (2010) Non-traumatic orbital hemorrhage. J Emerg Med 38(3):378-380. https://doi.org/10.1016/j.jemermed.2007.10.021

14. Pavone P, Polizzi A, Marino SD, Corsello G, Falsaperla R, Marino S, Ruggieri M (2020) West syndrome: a comprehensive review. Neurol Sci 22:1-6

\section{Publisher's Note}

Springer Nature remains neutral with regard to jurisdictional claims in published maps and institutional affiliations.

\section{Submit your manuscript to a SpringerOpen ${ }^{\circ}$ journal and benefit from:}

- Convenient online submission

- Rigorous peer review

- Open access: articles freely available online

- High visibility within the field

- Retaining the copyright to your article

Submit your next manuscript at $\boldsymbol{\nabla}$ springeropen.com 